\title{
ON THE WEAK MAXIMIZING PROPERTIES
}

\author{
LUIS C. GARCÍA-LIROLA AND COLIN PETITJEAN
}

\begin{abstract}
Quite recently, a new property related to norm-attaining operators has been introduced: the weak maximizing property (WMP). In this note, we define a generalised version of it considering other topologies than the weak one (mainly the weak* topology). We provide new sufficient conditions, based on the moduli of asymptotic uniform smoothness and convexity, which imply that a pair $(X, Y)$ enjoys a certain maximizing property. This approach not only allows us to (re)obtain as a direct consequence that the pair $\left(\ell_{p}, \ell_{q}\right)$ has the WMP, but also provides many more natural examples of pairs having a given maximizing property.
\end{abstract}

\section{INTRODUCTION}

Let $X, Y$ be two real Banach spaces and let $T: X \rightarrow Y$ be a bounded linear operator (we will write $T \in \mathcal{L}(X, Y)$ ). A maximizing sequence for $T$ is a sequence $\left(x_{n}\right)_{n} \subset X$ with $\left\|x_{n}\right\|=1$ for every $n \in \mathbb{N}$ and such that $\lim _{n \rightarrow \infty}\left\|T x_{n}\right\|_{Y}=\|T\|$. Next, we say as usual that $T: X \rightarrow Y$ attains its norm whenever there exists a vector $x \in X$ of norm 1 such that $\|T x\|_{Y}=\|T\|$. Now following [5], a pair of Banach spaces $(X, Y)$ is said to have the weak maximizing property (WMP) if for any bounded linear operator $T: X \rightarrow Y$, the existence of a non-weakly null maximizing sequence for $T$ implies that $T$ attains its norm. For instance, it is proved in [28, Theorem 1] that the pair $\left(\ell_{p}, \ell_{q}\right)$ has the WMP whenever $1<p<\infty$ and $1 \leq q<\infty$. That result was extended in [5, Proposition 2.2] to the pair $\left(\ell_{p}\left(\Gamma_{1}\right), \ell_{q}\left(\Gamma_{2}\right)\right)$ where $\Gamma_{1}, \Gamma_{2}$ are arbitrary indices.

Needless to say that the theory of norm attaining operators finds many applications in both pure and applied mathematics. A motivation for the study of the WMP is the following application which was noted in [5] (and extends a former result due to J. Kover in the Hilbert case [22]): if $(X, Y)$ has the weak maximizing property, $T \in \mathcal{L}(X, Y)$ and $K: X \rightarrow Y$ is a compact operator such that $\|T\|<\|T+K\|$, then $T+K$ is norm attaining. As a consequence, the authors could deduce that a pair $(X, Y)$ has the weak maximizing property for some $Y \neq\{0\}$ if and only if $X$ is reflexive.

In this note, we provide a new (possibly simpler) approach based on a comparison between the modulus of asymptotic uniform convexity of $X$ and the modulus of asymptotic uniform smoothness of $Y$, and conversely (see Section 2 for precise definitions). Moreover, we consider the following related and quite natural properties.

Definition 1.1. Let $X$ and $Y$ be Banach spaces. Let $\tau_{X}$ and $\tau_{Y}$ be any topology on $X$ and $Y$ respectively. We say that the pair:

- $\left(X^{*}, Y\right)$ has the weak* maximizing property $\left(\mathrm{W}^{*} \mathrm{MP}\right)$ if for any bounded linear operator $T: X^{*} \rightarrow Y$, the existence of a non-weak* null maximizing sequence for $T$ implies that $T$ attains its norm.

Date: July, 2020

2010 Mathematics Subject Classification. Primary 46B20; Secondary 46B04, 54E50.

Key words and phrases. Norm attainment, Banach Space, Weak Maximizing Property, Asymptotic Uniform Smoothness, Asymptotic Uniform Convexity. 
- $(X, Y)$ has the $\tau_{X}$-to- $\tau_{Y}$ maximizing property $\left(\tau_{X}\right.$-to- $\left.\tau_{Y} \mathrm{MP}\right)$ if for any bounded linear operator $T: X \rightarrow Y$ which is $\tau_{X}$-to- $\tau_{Y}$ continuous, the existence of a non $\tau_{X}$-null maximizing sequence for $T$ implies that $T$ attains its norm.

In this note, $\tau_{X}$ and $\tau_{Y}$ will mainly be the usual weak or weak* topologies. For instance, the WMP corresponds to the weak-to-weakMP. Notice that if the pair $\left(X^{*}, Y\right)$ has the WMP then it also has the $\mathrm{W}^{*} \mathrm{MP}$, and consequently the weak*to-weakMP. Also, if $X$ is reflexive, then a pair $(X, Y)$ has the WMP if and only if $(X, Y)$ has the $\mathrm{W}^{*} \mathrm{MP}$ if and only if $(X, Y)$ has the weak*-to-weakMP. However, we will prove that these three properties do not coincide in general.

Remark 1.2. The definition of the above properties can be stated in terms of nets instead of sequences. Indeed, given an operator $T: X \rightarrow Y$, the following properties are equivalent:

i) There exists a non $\tau_{X}$-null maximizing sequence for $T$.

ii) There exists a non $\tau_{X}$-null maximizing net for $T$.

iii) There exists a net which is maximizing for $T$ and does not admit 0 as a $\tau_{X}$-cluster point.

Clearly, i) $\Rightarrow$ ii). To see that iii $\Rightarrow$ i), let $\left(x_{\alpha}\right)_{\alpha} \subset S_{X}$ be a net such that 0 is not a $\tau_{X^{-}}$ cluster point and $\lim _{\alpha}\left\|T x_{\alpha}\right\|=\|T\|$. Pick inductively $\alpha_{n}$ such that $\left\|T x_{\alpha_{n}}\right\| \geq 1-\frac{1}{n}$ and $\alpha_{n} \geq \alpha_{m}$ if $n \geq m$. Then the sequence $\left(x_{\alpha_{n}}\right)_{n}$ is a subnet of $\left(x_{\alpha}\right)_{\alpha}$, so it is not $\tau_{X}$-null, and it is maximizing for $T$. Finally, assume that ii) holds and let's prove iii). Let $\left(x_{\alpha}\right)_{\alpha}$ be a non $\tau_{X}$-null net maximizing for $T$. Then 0 is not the only $\tau_{X}$-cluster point of $\left(x_{\alpha}\right)_{\alpha}$, otherwise, all the subnets of $\left(x_{\alpha}\right)_{\alpha}$ would converge to 0 , a contradiction (see e.g. [4]). Thus, there is a subnet $\left(y_{\beta}\right)_{\beta}$ of $\left(x_{\alpha}\right)_{\alpha}$ convergent to $y \neq 0$, and $\left(y_{\beta}\right)_{\beta}$ is also maximizing for $T$.

Remark 1.3. Replacing the WMP by the weak*-to-weak*MP, one can follow the lines of [5, Proposition 2.4] to obtain the following result:

Suppose that $\left(X^{*}, Y^{*}\right)$ has the weak*-to-weak* MP. Let $T, K: X^{*} \rightarrow Y^{*}$ be weak*to-weak* continuous linear operators such that $K$ is compact. If $\|T\|<\|T+K\|$ then $T+K$ is norm attaining.

We now describe the main findings of this paper. Throughout the paper, $X$ and $Y$ will denote real Banach spaces while $X^{*}$ denotes as usual the topological dual of $X$. After recalling the definition of the modulus $\bar{\delta}_{X^{*}}^{*}(t)$ of weak ${ }^{*}$ asymptotic uniform convexity and of the modulus $\bar{\rho}_{Y}(t)$ of asymptotic uniform smoothness in Section 2, we prove our first theorem in Section 3.

Theorem 3.1. Let $X, Y$ be Banach spaces. Assume that for every $t>0, \bar{\delta}_{X^{*}}^{*}(t) \geq$ $\bar{\rho}_{Y}(t)$ and, for all $t \geq 1$, either $\bar{\delta}_{X^{*}}^{*}(t)>\bar{\rho}_{Y}(t)$ or $\bar{\rho}_{Y}(t)>t-1$. Then,

(1) the pair $\left(X^{*}, Y\right)$ has the weak ${ }^{*}$-to-weakMP.

(2) If moreover $Y \equiv Z^{*}$ is a dual space, then the pair $\left(X^{*}, Z^{*}\right)$ has the weak*to-weak* $M P$.

(3) If $\bar{\delta}_{X^{*}}^{*}(t)=t$, then $\left(X^{*}, Y\right)$ has the $W^{*} M P$ for any Banach space $Y$. In particular, if $X$ is reflexive then the pair $\left(X^{*}, Y\right)$ has the WMP.

Since for any infinite sets $\Gamma_{1}$ and $\Gamma_{2}$ and for $1<p<q<\infty$, it is well known that $\bar{\delta}_{\ell_{p}\left(\Gamma_{1}\right)}^{*}(t)=\left(1+t^{p}\right)^{1 / p}-1>\left(1+t^{q}\right)^{1 / q}-1=\bar{\rho}_{\ell_{q}\left(\Gamma_{2}\right)}$, our last theorem provides a new proof of the fact that the pair $\left(\ell_{p}\left(\Gamma_{1}\right), \ell_{q}\left(\Gamma_{2}\right)\right)$ has the WMP. Note that the case $p=q$ also follows from the last theorem. In both papers [5, 28], the proof for the case $1 \leq q<p<\infty$ follows from Pitt's theorem asserting that any bounded operator $T: \ell_{p} \rightarrow \ell_{q}$ is compact and thus attains its norm. In fact, Pitt's theorem can also be generalised using the modulus $\bar{\rho}_{X}(t)$ of asymptotic uniform smoothness 
of $X$ and the modulus $\bar{\delta}_{Y}(t)$ of asymptotic uniform convexity of $Y$. Namely, if there exists $t>0$ such that $\bar{\rho}_{X}(t)<\bar{\delta}_{Y}(t)$, then every bounded linear operator from $X$ to $Y$ is compact (see [17, Proposition 2.3], where this is stated only for $0<t<1$, but the proof works for any $t>0)$. We take advantage of this fact to deduce the next result.

Theorem 3.8. Let $X, Y$ be Banach spaces and $|\cdot|$ be an equivalent norm on $X^{*}$. If there exists $t>0$ such that $\bar{\rho}_{|\cdot|}(t)<\bar{\delta}_{Y}(t)$, then

(1) the pair $\left(X^{*}, Y\right)$ has the weak ${ }^{*}$-to-weakMP.

(2) If moreover $Y \equiv Z^{*}$ is a dual spaces, then $\left(X^{*}, Z^{*}\right)$ has the weak*-toweak $^{*} M P$.

In particular, if $X$ is reflexive then $\left(X^{*}, Y\right)$ has the WMP.

In Section 4, we discuss the case of some classical Banach spaces and the connections between several weak maximizing properties. Notably, we show in Proposition 4.2 that if $X$ is reflexive, $Y$ has the Dunford-Pettis property and $(X, Y)$ has the WMP then $\left(Y^{*}, X^{*}\right)$ has the weak*-to-weak*MP. Next, we prove that Schur spaces such as $\ell_{1}$ are the best range spaces for the WMP: the pair $(X, Y)$ has the WMP for any reflexive space $X$ and any Schur space $Y$ (Proposition 4.7). It follows from Theorem $3.1(3)$ (and $\bar{\delta}_{\ell_{1}}^{*}(t)=t$ ) that $\ell_{1}$ is also a very good domain for the $\mathrm{W}^{*} \mathrm{MP}$ (see Corollary 4.8) while this is not the case of every Schur space. Indeed, in Example 4.9 (Example 4.10 respectively) we provide a dual Banach space $Z=X^{*}$ with the Schur property and such that the pair $\left(X^{*}, \mathbb{R}\right)$ fails the $\mathrm{W}^{*} \mathrm{MP}$ (the pair $\left(X^{*}, \ell_{1}\right)$ fails the weak ${ }^{*}$-to-weak ${ }^{*} \mathrm{MP}$ respectively). We end the paper by dealing with the classical James sequence spaces, Orlicz spaces and we also add some comments about the pair $\left(L_{p}([0,1]), L_{q}([0,1])\right)$.

\section{Preliminaries: ASYmptotic Uniform SMOOTHNESS AND CONVEXity}

Consider a real Banach space $(X,\|\cdot\|)$ and let $S_{X}$ be its unit sphere. For $t>0$, $x \in S_{X}$ we consider

$$
\begin{aligned}
& \bar{\delta}_{X}(t, x)=\sup _{\operatorname{dim}(X / Y)<\infty} \inf _{y \in S_{Y}}\|x+t y\|-1 \\
& \bar{\rho}_{X}(t, x)=\inf _{\operatorname{dim}(X / Y)<\infty} \sup _{y \in S_{Y}}\|x+t y\|-1 .
\end{aligned}
$$

The modulus of asymptotic uniform convexity of $X$ is given by

$$
\bar{\delta}_{X}(t)=\inf _{x \in S_{X}} \bar{\delta}_{X}(t, x)
$$

and modulus of asymptotic uniform smoothness of $X$ is given by

$$
\bar{\rho}_{X}(t)=\sup _{x \in S_{X}} \bar{\rho}_{X}(t, x) .
$$

The space $(X,\|\cdot\|)$ is said to be asymptotically uniformly convex (AUC for short) if $\bar{\delta}_{X}(t)>0$ for each $t>0$ and it is said to be asymptotically uniformly smooth (AUS for short) if $\lim _{t \rightarrow 0} t^{-1} \bar{\rho}_{X}(t)=0$. If $X$ is a dual space and we considered only weak* closed subspaces of $X$ then the corresponding modulus is denoted by $\bar{\delta}_{X}^{*}(t)$. The space $X$ is said to be weak $k^{*}$ asymptotically uniformly convex $\left(\mathrm{AUC}^{*}\right)$ if $\bar{\delta}_{X}^{*}(t)>0$ for each $t>0$. We refer the reader to [17] and the references therein for a detailed study of these properties.

Let $p, q \in[1, \infty)$. We say that $X$ is weak w $^{*}$-asymptotic uniformly convex (abbreviated by $p$-AUC ${ }^{*}$ ) if there exists $C>0$ so that $\bar{\delta}_{X}^{*}(t) \geq C t^{p}$ for all $t \in[0,1]$. Similarly, we say that $X$ is $q$-asymptotic uniformly smooth (abbreviated by $q$-AUS) if there exists $C>0$ so that $\bar{\rho}_{X}(t) \leq C t^{q}$ for all $t \in[0,1]$. Let us highlight that the following is proved in [12, Corollary 2.4]. 
Proposition 2.1. Let $X$ be a Banach space.

(i) Then \|\|$_{X}$ is AUS if and and only if \|\|$_{X^{*}}$ is $A U C^{*}$.

(ii) If $p \in(1, \infty]$ and $q \in[1, \infty)$ are conjugate exponents, then \|\|$_{X}$ is $p$-AUS if and and only if \|\|$_{X^{*}}$ is $q-A U C^{*}$.

It is worth mentioning that if $X \equiv Z^{*}$ and the dual norm $\|\cdot\|_{Z^{*}}$ is AUS, then $X$ must be reflexive (see for instance Proposition 2.6 in [9]). The following proposition is elementary.

Proposition 2.2. Let $X$ be a Banach space. For any $t>0$, any weakly null net $\left(x_{\alpha}\right)_{\alpha}$ in $X$ and any $x \in X \backslash\{0\}$ we have:

$$
\limsup _{\alpha}\left\|x+x_{\alpha}\right\| \leq\|x\|\left(1+\bar{\rho}_{X}\left(\frac{\lim \sup _{\alpha}\left\|x_{\alpha}\right\|}{\|x\|}\right)\right) .
$$

For any weak $k^{*}$-null net $\left(x_{\alpha}^{*}\right)_{\alpha} \subset X^{*}$ and for any $x^{*} \in X^{*} \backslash\{0\}$ we have

$$
\liminf _{\alpha}\left\|x^{*}+x_{\alpha}^{*}\right\| \geq\left\|x^{*}\right\|\left(1+\bar{\delta}_{X}^{*}\left(\frac{\liminf \inf _{\alpha}\left\|x_{\alpha}^{*}\right\|}{\left\|x^{*}\right\|}\right)\right) .
$$

Assume now that $\varphi:[0, \infty) \rightarrow[0, \infty)$ is an Orlicz function which is 1-Lipschitz and such that $\lim _{t \rightarrow \infty} \varphi(t) / t=1$. Consider for $(s, t) \in \mathbb{R}^{2}$,

$$
N_{2}^{\varphi}(s, t)= \begin{cases}|s|+|s| \varphi(|t| /|s|) & \text { if } s \neq 0 \\ |t| & \text { if } s=0\end{cases}
$$

The following is proved in [18] (see Lemma 4.3 and its preparation).

Lemma 2.3. The function $N_{2}^{\varphi}$ is an absolute (or lattice) norm on $\mathbb{R}^{2}$, meaning that $N_{2}^{\varphi}\left(s_{1}, s_{2}\right) \leq N_{2}^{\varphi}\left(t_{1}, t_{2}\right)$, whenever $\left|s_{i}\right| \leq\left|t_{i}\right|$ for all $i \leq 2$.

When $X$ is a Banach space, it is easy to see that $\bar{\rho}_{X}$ is a 1-Lipschitz Orlicz function such that $\lim _{t \rightarrow \infty} \bar{\rho}_{X}(t) / t=1$. Thus, one can consider the norm $N_{2}^{\bar{\rho}_{X}}$. We will use in the sequel the following reformulation of Proposition 2.2 in terms of the $\operatorname{norm} N_{2}^{\bar{\rho}_{X}}$.

Lemma 2.4. Let $X$ be a Banach space. If $\left(x_{\alpha}\right)_{\alpha} \subset X$ is weakly-null and $x \in X$, then

$$
\underset{\alpha}{\limsup }\left\|x+x_{\alpha}\right\| \leq N_{2}^{\bar{\rho}_{X}}\left(\|x\|, \limsup _{\alpha}\left\|x_{\alpha}\right\|\right)
$$

Proof. If $x=0$ there is nothing to do, so we may assume that $x \neq 0$. By application of Proposition 2.2 we see that

$$
\limsup _{\alpha}\left\|x+x_{\alpha}\right\| \leq\|x\|\left(1+\bar{\rho}_{X}\left(\frac{\limsup _{\alpha}\left\|x_{\alpha}\right\|}{\|x\|}\right)\right)=N_{2}^{\bar{\rho}_{X}}\left(\|x\|, \limsup _{\alpha}\left\|x_{\alpha}\right\|\right)
$$

Example 2.5. Let us recall the asymptotic moduli for some classical spaces, mostly taken from $[26]$.

- Let $1 \leq p<\infty$. If $X=\left(\sum_{n=1}^{\infty} E_{n}\right)_{p}$, where $\operatorname{dim}\left(E_{n}\right)<\infty$, then $\bar{\delta}_{X}(t)=$ $\bar{\rho}_{X}(t)=\left(1+t^{p}\right)^{1 / p}-1$ for all $t>0$.

- For any infinite set $\Gamma$ and $1 \leq p<\infty, \bar{\delta}_{\ell_{p}(\Gamma)}(t)=\bar{\rho}_{\ell_{p}(\Gamma)}(t)=\left(1+t^{p}\right)^{1 / p}-1$ for all $t>0$.

- For any infinite set $\Gamma, \bar{\delta}_{c_{0}(\Gamma)}(t)=\bar{\rho}_{c_{0}(\Gamma)}(t)=\max \{0, t-1\}$ for all $t>0$.

- Let $J$ be the James space. Then $\bar{\delta}_{J}(t)=\bar{\rho}_{J}(t)=\left(1+t^{2}\right)^{1 / 2}-1$ for all $t>0$. 
- If $X$ has a Schauder basis $\left(e_{n}\right)_{n=1}^{\infty}$ which satisfies a lower (resp. upper) $\ell_{p}$-estimate with constant one, then $\bar{\delta}_{X}(t) \geq\left(1+t^{p}\right)^{1 / p}-1\left(\right.$ resp. $\bar{\rho}_{X}(t) \leq$ $\left.\left(1+t^{p}\right)^{1 / p}-1\right)$. For instance, the $p$-convexified Tsirelson space $T^{p}$ (see [8]) and the Lorentz sequence space $d(w, p)$ satisfy an upper $\ell_{p}$-estimate with constant 1 [24, p. 177]. Moreover, $d(w, p)$ contains almost isometric copies of $\ell_{p}$ so $\bar{\rho}_{d(w, p)}(t)=\left(1+t^{p}\right)^{1 / p}-1$ for all $t>0$.

\section{Proof of the Main Results}

Let us begin with the proof of our main theorem.

Theorem 3.1. Let $X, Y$ be Banach spaces. Assume that for every $t>0, \bar{\delta}_{X^{*}}^{*}(t) \geq$ $\bar{\rho}_{Y}(t)$ and, for all $t \geq 1$, either $\bar{\delta}_{X^{*}}^{*}(t)>\bar{\rho}_{Y}(t)$ or $\bar{\rho}_{Y}(t)>t-1$. Then,

(1) the pair $\left(X^{*}, Y\right)$ has the weak ${ }^{*}$-to-weakMP.

(2) If moreover $Y \equiv Z^{*}$ is a dual space, then the pair $\left(X^{*}, Z^{*}\right)$ has the weak ${ }^{*}$ to-weak* $M P$.

(3) If $\bar{\delta}_{X^{*}}^{*}(t)=t$ then $\left(X^{*}, Y\right)$ has the $W^{*} M P$ for any Banach space $Y$.

In particular, if $X$ is reflexive then the pair $\left(X^{*}, Y\right)$ has the WMP.

Proof. We will only prove the first assertion since the proof of the second and third one are very similar (replacing the weak topology in $Y$ by the weak* topology in $Y$ ).

Let $T: X^{*} \rightarrow Y$ be a bounded operator which is weak*-to-weak continuous. Without loss of generality, we may assume that $T$ has norm 1 . Let $\left(x_{n}\right)$ be a normalized maximizing sequence in $X^{*}$ which is not weak ${ }^{*}$-null. Let $\left(x_{\alpha}\right)$ be a subnet which is weak ${ }^{*}$ convergent to $x \neq 0$. By extracting a subnet again, we may assume that $\lim _{\alpha}\left\|x_{\alpha}-x\right\|$ and $\lim _{\alpha}\left\|T x-T x_{\alpha}\right\|$ exists. Since $T$ is weak*-to-weak continuous, we have that $T x_{\alpha} \stackrel{w}{\longrightarrow} T x$. Using Lemma 2.4, we thus have the following estimates:

$$
\begin{aligned}
1 & =\|T\|=\lim _{\alpha}\left\|T x_{\alpha}\right\|=\lim _{\alpha}\left\|T x+T x_{\alpha}-T x\right\| \\
& \leq N_{2}^{\bar{\rho}_{Y}}\left(\|T x\|, \lim _{\alpha}\left\|T x_{\alpha}-T x\right\|\right) .
\end{aligned}
$$

Now notice that (we use Lemma 2.3)

$$
\begin{array}{r}
N_{2}^{\bar{\rho}_{Y}}\left(\|T x\|, \lim _{\alpha}\left\|T x_{\alpha}-T x\right\|\right) \leq N_{2}^{\bar{\rho}_{Y}}\left(\|x\|, \lim _{\alpha}\left\|T x_{\alpha}-T x\right\|\right) \\
\leq N_{2}^{\bar{\rho}_{Y}}\left(\|x\|, \lim _{\alpha}\left\|x_{\alpha}-x\right\|\right)
\end{array}
$$

but also

$$
\begin{array}{r}
N_{2}^{\bar{\rho}_{Y}}\left(\|T x\|, \lim _{\alpha}\left\|T x_{\alpha}-T x\right\|\right) \leq N_{2}^{\bar{\rho}_{Y}}\left(\|T x\|, \lim _{\alpha}\left\|x_{\alpha}-x\right\|\right) \\
\leq N_{2}^{\bar{\rho}_{Y}}\left(\|x\|, \lim _{\alpha}\left\|x_{\alpha}-x\right\|\right) .
\end{array}
$$

Since $x \neq 0$, we deduce from the definition of $N_{2}^{\bar{\rho}_{Y}}$ the following estimates:

$$
\begin{aligned}
N_{2}^{\bar{\rho}_{Y}}\left(\|x\|, \lim _{\alpha}\left\|x_{\alpha}-x\right\|\right) & \leq\|x\|+\|x\| \bar{\rho}_{Y}\left(\frac{\lim _{\alpha}\left\|x_{\alpha}-x\right\|}{\|x\|}\right) \\
& \leq\|x\|+\|x\| \bar{\delta}_{X^{*}}^{*}\left(\frac{\lim _{\alpha}\left\|x_{\alpha}-x\right\|}{\|x\|}\right) \\
& \leq \lim _{\alpha}\left\|x+x_{\alpha}-x\right\| \\
& =1 .
\end{aligned}
$$

This implies that all the previous inequalities are in fact equalities, in particular

$$
N_{2}^{\bar{\rho}_{Y}}\left(\|T x\|, \lim _{\alpha}\left\|x_{\alpha}-x\right\|\right)=N_{2}^{\bar{\rho}_{Y}}\left(\|x\|, \lim _{\alpha}\left\|x_{\alpha}-x\right\|\right)=1=N_{2}^{\bar{\rho}_{Y}}(0,1) .
$$


This means that the points $\left(\|T x\|, \lim _{\alpha}\left\|x_{\alpha}-x\right\|\right),\left(\|x\|, \lim _{\alpha}\left\|x_{\alpha}-x\right\|\right)$ and $(0,1)$ are aligned in $\mathbb{R}^{2}$. If $\|T x\|=\|x\|$ or $\lim _{\alpha}\left\|x-x_{\alpha}\right\|=0$, then $T$ attains its norm at $x$ and we are done. Otherwise, it follows that $\lim _{\alpha}\left\|x_{\alpha}-x\right\|=1$ and

$$
1=N_{2}^{\bar{\rho}_{Y}}(\|x\|, 1)=\|x\|+\|x\| \bar{\rho}_{Y}(1 /\|x\|)=\|x\|+\|x\| \bar{\delta}_{X^{*}}^{*}(1 /\|x\|) .
$$

That is, $\bar{\delta}_{X^{*}}\left(\frac{1}{\|x\|}\right)=\bar{\rho}_{Y}\left(\frac{1}{\|x\|}\right)=\frac{1}{\|x\|}-1$, which contradicts our assumptions.

Remark 3.2. Take $X=\mathbb{R} \oplus_{\infty} \ell_{2}$ and $Y=c_{0}$. Then $\bar{\delta}_{X}(t)=\max \{0, t-1\}=\bar{\rho}_{Y}(t)$ for all $t>0$. However, the operator $T: X \rightarrow Y$ given by $T\left(\left(0, e_{n}\right)\right)=\frac{n}{n+1} e_{n}$ and $T((1,0))=0$ does not attain the norm and admits the non-weakly null maximizing sequence $\left(x_{n}\right)_{n=1}^{\infty}$ given by $x_{n}=\left(1, e_{n}\right)$. Thus, the pair $(X, Y)$ fails the WMP.

Remark 3.3. Since $\bar{\rho}_{Y}$ is 1-Lipschitz and $\bar{\rho}_{Y}(t) \leq \max \{0, t-1\}$, the condition $\bar{\rho}_{Y}(1)=0$ is equivalent to $\bar{\rho}_{Y}(t)=\max \{0, t-1\}$ for all $t>0$. These spaces are called metric weak ${ }^{*}$ Kadec-Klee spaces. In the separable case, they are precisely those spaces which are $(1+\varepsilon)$-isomorphic to a subspace of $c_{0}$, for every $\varepsilon>0$ [15].

As a direct consequence we obtain that for a special class of Banach spaces, namely the dual ones $X^{*}$ with $S z(X)=\omega$ (where $\omega$ stands for the first countable ordinal), one can find an equivalent norm $|\cdot|$ on $X^{*}$ and a Banach space $Y$ such that the pair $\left(\left(X^{*},|\cdot|\right), Y\right)$ has the weak*-to-weak*MP. By $S z(X)$ we mean the Szlenk index of $X$; we refer to [23] for its definition, basic properties as well as its main applications to the geometry of Banach spaces.

Corollary 3.4. If $X$ is separable with $S z(X)=\omega$, then there exists an equivalent norm $|\cdot|_{X^{*}}$ on $X^{*}$ and $q \geq 1$ such that the pair $\left(\left(X^{*},|\cdot| X^{*}\right), \ell_{q}\right)$ has the weak $k^{*}$-toweak ${ }^{*} M P$.

In particular if $X \equiv Z^{*}$ is a separable reflexive space with $S z(Z) \leq \omega$, then there exists an equivalent norm on $X$ and there exists $q \geq 1$ such that the pair $\left(X, \ell_{q}\right)$ has the WMP.

Proof. Let us start by recalling the fundamental renorming result for spaces with Szlenk index equal to $\omega$. The result is due to H. Knaust, E. Odell and Th. Schlumprecht [21, Corollary 5.3]: if $X$ is a separable Banach space such that $S z(X)=\omega$, then there exists $p \in(1, \infty)$ such that $X^{*}$ admits an equivalent $p$-AUC* norm $|\cdot|$, moreover $\bar{\delta}_{|\cdot|}^{*}(t) \geq\left(1+t^{p}\right)^{\frac{1}{p}}-1$. Let us consider $q>p$. As we already mentioned in Example 2.5, $\bar{\rho}_{\ell_{q}}(t)=\left(1+t^{q}\right)^{\frac{1}{q}}-1$. Thus for every $t>0$ we have that $\bar{\delta}_{|\cdot|}^{*}(t)>\bar{\rho}_{\ell_{q}}(t)$. The result now readily follows from Theorem 3.1.

The next result follows the idea of [28, Theorem 2], where the same is obtained in the case $X^{*}=\ell_{p}, Y=\ell_{q}, p \neq q$.

Corollary 3.5. Let $X, Y$ be Banach spaces. Assume that $X$ is separable and $\bar{\delta}_{X^{*}}^{*}(t)>\bar{\rho}_{Y}(t)$ for all $t>0$. Let $T: X^{*} \rightarrow Y$ be a weak $k^{*}$-to-weak continuous operator. Then any non-weak* null maximizing sequence for $T$ has a convergent subsequence.

Proof. Let $\left(x_{n}\right)_{n=1}^{\infty} \subset S_{X^{*}}$ be a non-weak* null maximizing sequence for $T$. By extracting a subsequence, we may assume that $\left(x_{n}\right)$ is weak* convergent to $x \neq 0$ and the $\operatorname{limit}_{n}\left\|\lim _{n}-x\right\|$ exists. The proof of Theorem 3.1 shows that $T$ attains its norm at $x$, and moreover

$$
\bar{\rho}_{Y}\left(\frac{\lim _{n}\left\|x_{n}-x\right\|}{\|x\|}\right)=\bar{\delta}_{X^{*}}^{*}\left(\frac{\lim _{n}\left\|x_{n}-x\right\|}{\|x\|}\right) .
$$

Thus $\lim _{n}\left\|x_{n}-x\right\|=0$. 
We will now turn to the case when the modulus of asymptotic uniform smoothness of $X$ is bounded above by the modulus of asymptotic uniform convexity of $Y$. To this aim, we will need the following two lemmata.

Lemma 3.6 (Proposition 2.3 in [17]). Let $X, Y$ be Banach spaces. If there exists $t>0$ such that $\bar{\rho}_{X}(t)<\bar{\delta}_{Y}(t)$ then every linear operator from $X$ to $Y$ is compact.

The next one is classical and easy to prove.

Lemma 3.7. Let $X, Y$ be Banach spaces and let $\tau_{Y}$ be any Hausdorff topology on $Y$ which is coarser than the norm topology. If $T: X^{*} \rightarrow Y$ is a compact operator which is weak*-to- $\tau_{Y}$ continuous, then $T$ attains its norm.

Proof. Let $\left(x_{\alpha}^{*}\right)$ be a net in $S_{X^{*}}$ such that $\lim _{\alpha}\left\|T x_{\alpha}^{*}\right\|=\|T\|$, we may assume that $\left(x_{\alpha}^{*}\right)$ is weak*-convergent to $x^{*} \in B_{X^{*}}$. Then $\left(T x_{\alpha}^{*}\right)$ is $\tau_{Y^{-}}$-convergent to $T x^{*}$. Since the norm topology and $\tau_{Y}$ agree on the norm-compact set $\overline{T\left(B_{X^{*}}\right)}$, we have that $\|T\|=\lim _{\alpha}\left\|T x_{\alpha}^{*}\right\|=\left\|T x^{*}\right\|$.

We are now ready to prove the desired theorem.

Theorem 3.8. Let $X, Y$ be Banach spaces and $|\cdot|$ be an equivalent norm on $X^{*}$. If there exists $t>0$ such that $\bar{\rho}_{|\cdot|}(t)<\bar{\delta}_{Y}(t)$, then

(1) the pair $\left(X^{*}, Y\right)$ has the weak ${ }^{*}$-to-weakMP.

(2) If moreover $Y \equiv Z^{*}$ is a dual space, then $\left(X^{*}, Z^{*}\right)$ has the weak*-toweak $k^{*}$ P.

In particular, if $X$ is reflexive then $\left(X^{*}, Y\right)$ has the WMP.

Proof. We only prove the first assertion, the proof of the second one is similar and the last statement is quite obvious. Thanks to Lemma 3.6, we know that every bounded linear operator from $\left(X^{*},|\cdot|\right)$ to $Y$ is compact. Since $|\cdot|$ is an equivalent norm on $X^{*}$, this readily implies that every bounded linear operator from $X^{*}$ to $Y$ is compact. According to Lemma 3.7, we deduce that every weak*-to-weak continuous operator from $X^{*}$ to $Y$ attains its norm.

We highlight some consequences of the previous theorems for particular spaces. In all the cases the proof follows from Theorem 3.1 or Theorem 3.8 and Example 2.5.

Corollary 3.9. Any of the following conditions ensues that the pair $(X, Y)$ has the $W M P$ :

a) $X$ is a reflexive AUS space (e.g. $X=\ell_{p}, T^{p}$, or $\left.d(w, p), 1<p<\infty\right)$, and $Y=\ell_{1}$.

b) $X$ is a reflexive $A U C$ space (e.g. $X=\ell_{p}$ ) and $Y=c_{0}$.

c) $X=\ell_{p}$, and either $Y=\ell_{q}, 1<p, q<\infty$, or $Y=J$, the James space.

d) $X=\ell_{p}$ and either $Y=T^{q}$ or $Y=d(w, q)$ where $1<p \leq q<\infty$.

\section{Application to some classical Banach spaces}

4.1. Dunford-Pettis spaces. We recall that a Banach space $Y$ has the DunfordPettis property if and only if for every sequence $\left(y_{n}\right)_{n}$ in $Y$ converging weakly to 0 and every sequence $\left(y_{n}^{*}\right)_{n}$ in $Y^{*}$ converging weakly to 0 , the sequence of scalars $\left(y_{n}^{*}\left(y_{n}\right)\right)_{n}$ converges to 0 . In particular, Schur spaces have it. Classical spaces with the Dunford-Pettis property include as $c_{0}, \mathcal{C}(K)$ spaces (where $K$ is a compact Hausdorff space) and $L_{1}(\mu)$ (where $\mu$ is a $\sigma$-finite measure), see e.g. [11]. However reflexive spaces never have it.

Lemma 4.1. Assume that $X$ is reflexive and that $Y$ has the Dunford-Pettis property. Let $T: X \rightarrow Y$ be a bounded operator. Then the following are equivalent:

(i) There exists a non-weakly null maximizing sequence for $T$. 
(ii) There exists a non-weakly null maximizing sequence for $T^{*}$.

Proof. First of all, if $\left(x_{n}\right)_{n} \subset X$ is a maximizing sequence for $T$, we define $\left(y_{n}^{*}\right)_{n} \subset$ $S_{Y^{*}}$ so that $y_{n}^{*}\left(T x_{n}\right)>\left\|T x_{n}\right\|-\frac{1}{n}$. It is easy to see that

$$
\left\|T^{*} y_{n}^{*}\right\| \geq\left|y_{n}^{*}\left(T x_{n}\right)\right|>\left\|T x_{n}\right\|-\frac{1}{n}
$$

and taking the limit $n \rightarrow \infty$ we obtain $\lim _{n \rightarrow \infty}\left\|T^{*} y_{n}^{*}\right\| \geq\|T\|$. Since $\left\|T^{*}\right\|=\|T\|$ we deduce that $\left(y_{n}^{*}\right)_{n}$ is a maximizing sequence for $T^{*}$. We use the exact same argument to build a maximizing sequence for $T$ starting from one for $T^{*}$.

Now let $T: X \rightarrow Y$ be an operator and let $\left(x_{n}\right)_{n}$ be a non-weakly null maximizing sequence for $T$. By weak-compactness, we may extract a subsequence (still denoted the same way) which weakly converges to some $x$. Now as $T$ is weakly continuous, we have that $\left(T x_{n}\right)$ weakly converges to $T x$. Let $\left(y_{n}^{*}\right)_{n}$ be the sequence constructed above. We claim that $\left(y_{n}^{*}\right)_{n}$ is not weakly null. Indeed, aiming for a contradiction, assume that $\left(y_{n}^{*}\right)_{n}$ is weakly null. As $Y$ has the Dunford-Pettis property, we have that $\lim _{n \rightarrow \infty} y_{n}^{*}\left(T x_{n}\right)=\lim _{n \rightarrow \infty} y_{n}^{*}\left(T x_{n}-T x\right)=0$, which contradicts our definition of $\left(y_{n}^{*}\right)_{n}$.

The converse is proved in the same way.

Proposition 4.2. Let $X$ be a reflexive space and let $Y$ be a Banach space with the Dunford-Pettis property. If $(X, Y)$ has the WMP then any dual operator $T^{*}: Y^{*} \rightarrow$ $X^{*}$ which admits a non-weakly null maximizing sequence attains its norm. In particular the pair $\left(Y^{*}, X^{*}\right)$ has the weak ${ }^{*}$-to-weak $k^{*} M P$.

Proof. let $T^{*}: Y^{*} \rightarrow X^{*}$ be the dual operator of a bounded operator $T: X \rightarrow Y$. Let $\left(y_{n}^{*}\right)_{n}$ be a non-weakly null maximizing sequence for $T$. According to Lemma 4.1, $T$ admits a non-weakly null maximizing sequence. Since $(X, Y)$ has the WMP, $T$ attains its norm at some $x$. Now using Hahn-Banach theorem, we may pick $y^{*} \in Y^{*}$ such that $y^{*}(T x)=\|T x\|=\|T\|$. It is readily seen that the operator $T^{*}$ attains its norm at $y^{*}$.

Note that the hypothesis " $X$ is reflexive" in the previous proposition is actually superfluous as the assumption " $(X, Y)$ has the WMP" already implies that $X$ must be reflexive [5].

We do not know whether the hypothesis on the Dunford-Pettis property is actually needed in the previous result. More precisely:

Question 4.3. Assume that $(X, Y)$ has the WMP. Does it follow that $\left(Y^{*}, X^{*}\right)$ has the weak*-to-weak* MP?

On the other hand, there are spaces $X, Y$ so that $\left(Y^{*}, X^{*}\right)$ has the WMP but $(X, Y)$ fails the WMP: just take $Y=\mathbb{R}$ and $X$ any non-reflexive space. We do not know if it is possible to construct such an example in the reflexive case, that is:

Question 4.4. Let $X, Y$ be reflexive spaces so that $\left(Y^{*}, X^{*}\right)$ has the WMP. Does it follow that $(X, Y)$ has the WMP?

Notice that, under the same conditions as in Proposition 4.2, $(X, Y)$ having the WMP does not imply that $\left(X^{* *}, Y^{* *}\right)$ has the weak ${ }^{*}$-to-weak ${ }^{*} \mathrm{MP}$ (while $\left(Y^{*}, X^{*}\right.$ ) does so). Indeed, the next example shows that $\left(\ell_{2}, c_{0}\right)$ has the WMP but $\left(\ell_{2}, \ell_{\infty}\right)$ fails the weak*-to-weak* MP. It was also suggested to us by R. Aron to consider the following variation of the WMP:

Definition 4.5. We say that a pair $(X, Y)$ has the bidual WMP if for every operator $T: X \rightarrow Y$, the existence of a non weakly-null maximizing sequence for $T$ implies that $T^{* *}: X^{* *} \rightarrow Y^{* *}$ attains its norm. 
It is obvious from the definitions that if $\left(X^{* *}, Y^{* *}\right)$ has the weak ${ }^{*}$-to-weak ${ }^{*} \mathrm{MP}$ then $(X, Y)$ has the bidual WMP. However, the next example shows in particular that the converse does not hold in general.

Example 4.6. First of all, notice that $\left(\ell_{2}, c_{0}\right)$ has the WMP thanks to Corollary $3.9 \mathrm{~b}$ ), thus it also has the bidual WMP. Moreover, $\ell_{2}$ is reflexive and $c_{0}$ has the Dunford-Pettis property so that Proposition 4.2 implies that $\left(\ell_{1}, \ell_{2}\right)$ has the weak*-to-weak* MP (even the $\mathrm{W}^{*} \mathrm{MP}$, see Corollary 4.8). Next, we will prove that $\left(\ell_{2}, \ell_{\infty}\right)$ fails the weak*-to-weak* MP (and consequently also fails the WMP and the $\left.\mathrm{W}^{*} \mathrm{MP}\right)$.

Let $T: \ell_{2} \rightarrow \ell_{\infty}$ defined for every $x=\left(x_{n}\right)_{n} \in \ell_{2}$ by

$$
T x=\left(x_{1}, x_{1}+\left(1-\frac{1}{2}\right) x_{2}, x_{1}+\left(1-\frac{1}{3}\right) x_{3}, \ldots, x_{1}+\left(1-\frac{1}{n}\right) x_{n}, \ldots\right) .
$$

In other words, $T e_{1}=\mathbb{1}$ (the constant equal to 1 sequence) and $T e_{n}=\left(1-\frac{1}{n}\right) e_{n}$. Our claim follows from the items bellow:

- $\|T\|=\sqrt{2}$. Indeed,

$$
\begin{aligned}
\|T x\|_{\infty} & =\sup \left\{\left|x_{1}+(1-1 / n) x_{n}\right|: n \geq 1\right\} \\
& \leq \sup \left\{\left(\left|x_{1}\right|^{2}+\left|x_{n}\right|^{2}\right)^{1 / 2}\left(1+(1-1 / n)^{2}\right)^{1 / 2}: n \geq 2\right\} \\
& \leq \sqrt{2}\|x\|_{2} .
\end{aligned}
$$

Moreover,

$$
\left\|T\left(\frac{1}{\sqrt{2}}\left(e_{1}+e_{n}\right)\right)\right\| \rightarrow \sqrt{2},
$$

so $\|T\|=\sqrt{2}$ the sequence $\left(\frac{1}{\sqrt{2}}\left(e_{1}+e_{n}\right)\right)_{n}$ is a normalized maximizing sequence which is not weakly* null (it weakly* converges to $\frac{1}{\sqrt{2}} e_{1}$ ).

- $T$ is weak*-to-weak*-continuous: one can easily chech that $T=S^{*}$ where $S: \ell_{1} \rightarrow \ell_{2}$ is given by $S x=\left(\sum_{n=1}^{\infty} x_{n}\right) e_{1}+\sum_{n=1}^{\infty}(1-1 / n) x_{n} e_{n}$.

- $T$ does not attain its norm: if $\|T x\|_{\infty}=\sqrt{2}$ for some $x$ with $\|x\|_{2}=1$, then the above estimation implies that $\lim \sup _{n}\left(\left|x_{1}\right|^{2}+\left|x_{n}\right|^{2}\right)^{1 / 2}=1$. Thus $\left|x_{1}\right|=1$, which implies that $x= \pm e_{1}$, a contradiction.

4.2. The case of $\ell_{1}$ and Schur spaces. Recall that a Banach space $Y$ is said to have the Schur property if every weakly convergent sequence is also norm-convergent. First of all, let us point out that Schur spaces are the best range spaces for the WMP as this is shown by the next result.

Proposition 4.7. If $X$ is reflexive and $Y$ is Schur, then $(X, Y)$ has the WMP.

Proof. Let $T: X \rightarrow Y$ be an operator and let $\left(x_{n}\right)_{n}$ be a non-weakly null maximizing sequence for $T$. By weak-compactness, we may extract a subsequence (still denoted the same way) which weakly converges to some $x \in B_{X}$. Since $T$ is weakly continuous, $\left(T x_{n}\right)_{n}$ weakly converges to $T x$. Since $Y$ has the Schur property, $\left(T x_{n}\right)_{n}$ actually converges to $T x$ in the norm topology. The fact that $\lim _{n \rightarrow \infty}\left\|T x_{n}\right\|=\|T\|$ readily implies that $\|T x\|=\|T\|$.

It is well known that $\ell_{1} \equiv c_{0}^{*}$ is weak* asymptotically uniformly convex with modulus $\bar{\delta}_{\ell_{1}}^{*}(t)=t$, and the same is true for $Z^{*}$ instead of $\ell_{1}$ whenever $Z$ is a subspace of $c_{0}$. Consequently, the next result is a direct consequence of Theorem 3.1. Let us mention that if a dual Banach space $X \equiv Z^{*}$ has modulus $\bar{\delta}_{X}^{*}(t)=t$, then $Z$ is asymptotically uniformly flat (that is there exists $t_{0}>0$ such that for every 
$\left.t<t_{0}, \bar{\rho}_{Z}(t)=0\right)$. This is actually equivalent to the fact that $Z$ is isomorphic to a subspace of $c_{0}$ (see [17, Theorem 2.9]).

Corollary 4.8. If $Z$ is a closed subspace of $c_{0}$, then the pair $\left(Z^{*}, Y\right)$ has the $W^{*} M P$ for every Banach space $Y$.

In particular $\left(\ell_{1}, Y\right)$ has the $W^{*} M P$ for every Banach space $Y$.

However, the previous result is not true anymore if one replaces $\ell_{1}$ by any dual space with the Schur property as this is shown by the following example. It also shows that the $\mathrm{W}^{*} \mathrm{MP}$ is not an isomorphic property.

Example 4.9. There exists a dual space $Z=X^{*}$ with the Schur property such that the pair $\left(X^{*}, \mathbb{R}\right)$ fails the $\mathrm{W}^{*} \mathrm{MP}$. In fact $Z$ is going to be the Lipschitz-free space $\mathcal{F}(M)$ where $M=\{0\} \cup\left\{x_{n}: n \in \mathbb{N}\right\} \subset c_{0}$ is the pointed metric space given by $x_{1}=2 e_{1}$, and $x_{n}=e_{1}+\left(1+\frac{1}{n}\right) e_{n}$ for $n \geq 2$, where $\left(e_{n}\right)$ is the canonical basis of $c_{0}$. Then it is known [19] that $\mathcal{F}(M)$ has the Schur property as $M$ is uniformly discrete and bounded (it is actually isomorphic to $\ell_{1}$ ) and it is also known [14, Example 5.6] that $\mathcal{F}(M) \equiv X^{*}$ where

$$
X=\left\{f \in \operatorname{Lip}_{0}(M): \lim _{n \rightarrow \infty} f\left(x_{n}\right)=\frac{1}{2} f\left(x_{1}\right)\right\} .
$$

It is readily seen that the sequence $\left(\left(1+\frac{1}{n}\right)^{-1} \delta\left(x_{n}\right)\right)_{n}$ weak* converges to $\frac{1}{2} \delta\left(x_{1}\right) \neq 0$. Now let $f: M \rightarrow \mathbb{R}$ be the Lipschitz map defined by $f(0)=f\left(x_{1}\right)=0$ and $f\left(x_{n}\right)=1$ for every $n \geq 2$. It is obvious that $\|f\|_{L}=\lim _{n \rightarrow \infty} \frac{f\left(x_{n}\right)-f(0)}{d\left(x_{n}, 0\right)}=1$. Now using the linearization property of free spaces, we may consider the bounded operator $\bar{f}: \mathcal{F}(M) \rightarrow \mathbb{R}$ such that $\bar{f}\left(\delta\left(x_{n}\right)\right)=f\left(x_{n}\right)$. Observe that $\left(\left(1+\frac{1}{n}\right)^{-1} \delta\left(x_{n}\right)\right)_{n}$ is a maximizing sequence which is not weak ${ }^{*}$-null. To finish, we just need to show that $\bar{f}$ does not attain its norm. Assume that $\bar{f}(\mu)=1$ for $\mu=\sum_{n=1}^{\infty} a_{n} \delta\left(x_{n}\right) \in S_{\mathcal{F}(M)}$. First note that

$$
1=\bar{f}(\mu)=\sum_{n=2}^{\infty} a_{n}
$$

Let $A=\left\{n \geq 2: a_{n}>0\right\}$ and consider the one-Lipschitz function $g: M \rightarrow \mathbb{R}$ given by $g\left(x_{n}\right)=1$ if $n \in A$ and $g\left(x_{n}\right)=0$ otherwise. By evaluating the linear extension of $g$ to $\mathcal{F}(M)$ at $\mu$ we get $\sum_{n \in A} a_{n} \leq 1$. It follows that $a_{n} \geq 0$ for all $n \geq 2$. Similarly, by evaluating at the one-Lipschitz function $h: M \rightarrow \mathbb{R}$ given by $h\left(x_{1}\right)=2$ and $h\left(x_{n}\right)=1$ we get that $-1 \leq a_{1} \leq 0$. Now, we have that

$$
\mu=\sum_{n=1}^{\infty} a_{n} \delta\left(x_{n}\right)=\sum_{n=2}^{\infty} a_{n}\left|a_{1}\right|\left(\delta\left(x_{n}\right)-\delta\left(x_{1}\right)\right)+\sum_{n=1}^{\infty}\left(1-\left|a_{1}\right|\right) a_{n} \delta\left(x_{n}\right) .
$$

Finally, consider the one-Lipschitz function $j: M \rightarrow \mathbb{R}$ given by $j(0)=j\left(x_{1}\right)=0$ and $j\left(x_{n}\right)=1+1 / n$. Then,

$$
\begin{aligned}
1 & =\|\mu\| \geq\langle\bar{j}, \mu\rangle=\sum_{n=2}^{\infty} a_{n}\left|a_{1}\right|\left(1+\frac{1}{n}\right)+\sum_{n=2}^{\infty}\left(1-\left|a_{1}\right|\right) a_{n}\left(1+\frac{1}{n}\right) \\
& =\sum_{n=2}^{\infty} a_{n}\left(1+\frac{1}{n}\right)=1+\sum_{n=2}^{\infty} \frac{a_{n}}{n} .
\end{aligned}
$$

Thus, $a_{n}=0$ for all $n \geq 2$. Then $\bar{f}(\mu)=0$, a contradiction.

Notice that the Banach space $\mathcal{F}(M)$ in the previous example is not weak*-AUC: $\frac{1}{2} \delta\left(x_{1}\right)$ is an extreme point of $B_{\mathcal{F}(M)}$ [3] but not a preserved extreme point [2], in particular it does not belong to slices of small diameter.

The following example shows that the weak*-to-weak* MP is not stable by isomorphisms. 
Example 4.10. There exists a space $X$ isomorphic to $c_{0}$ such that $\left(X^{*}, \ell_{1}\right)$ fails the weak*-to-weak*MP. Indeed, let $M$ be the metric space given by $M=\mathbb{N} \cup\{0\}$ where $d(n, 0)=d(n, 1)=1+1 / n$ and $d(n, m)=2+1 / n+1 / m$ for all $n, m \geq 2$. The same argument as $\left[14\right.$, Example 5.6] gives that $\mathcal{F}(M) \equiv X^{*}$, where

$$
X=\left\{f \in \operatorname{Lip}_{0}(M): \lim _{n \rightarrow \infty} f\left(x_{n}\right)=\frac{1}{2} f\left(x_{1}\right)\right\} .
$$

It is easy to check that the map $T: c_{0} \rightarrow X$ given by $T x(1)=x_{1}$ and $T x(n)=\frac{1}{2}\left(x_{1}+\right.$ $x_{n}$ ) for $n \geq 2$ satisfies that $\|T\| \leq 1$. Moreover, $T$ is an isomorphism with inverse $R: X \rightarrow c_{0}$ given by $R f=f(1) e_{1}+\sum_{n \geq 2}(2 f(n)-f(1)) e_{n}$. Consider the weak*-toweak*-continuous operator $T^{*}: \mathcal{F}(M) \rightarrow \ell_{1}$. First note that $T^{*}(\delta(n))=\frac{1}{2}\left(e_{1}+e_{n}\right)$ for $n \geq 2$. Thus, $\left\|T^{*}\right\|=1$ and the sequence $\left(\left(1+\frac{1}{n}\right)^{-1} \delta(n)\right)_{n}$ is a maximizing sequence for $T^{*}$ which weak ${ }^{*}$-converges to $\frac{1}{2} \delta(1) \neq 0$. To finish, we need to check that $T^{*}$ does not attain its norm. Assume that there is $\mu=\sum_{n=1}^{\infty} a_{n} \delta\left(x_{n}\right) \in S_{\mathcal{F}(M)}$ is so that $\left\|T^{*} \mu\right\|_{1}=1$. Consider the 1-Lipschitz function $f: M \rightarrow \mathbb{R}$ given by $f(0)=f(1)=0$ and $f(n)=\left(1+\frac{1}{n}\right) \operatorname{sign}\left(a_{n}\right)$ for $n \geq 2$. Then

$$
1=\|\mu\| \geq\langle\bar{f}, \mu\rangle=\sum_{n \geq 2}\left|a_{n}\right|\left(1+\frac{1}{n}\right) .
$$

Now, consider the 1-Lipschitz function $g: M \rightarrow \mathbb{R}$ given by $g(0)=0, g(1)=2$ and $g(n)=1$ for $n \geq 2$. Then

$$
1=\|\mu\| \geq|\langle\bar{f}, \mu\rangle|=\left|2 a_{1}+\sum_{n \geq 2} a_{n}\right| .
$$

Note that

$$
T^{*} \mu=\left(a_{1}+\frac{1}{2} \sum_{n \geq 2} a_{n}\right) e_{1}+\frac{1}{2} a_{n} e_{n}
$$

and so

$$
1=\left\|T^{*} \mu\right\|_{1}=\left|a_{1}+\frac{1}{2} \sum_{n \geq 2} a_{n}\right|+\frac{1}{2} \sum_{n \geq 2}\left|a_{n}\right| \leq 1 .
$$

It follows that $\sum_{n \geq 2}\left|a_{n}\right|=1 \geq \sum_{n \geq 2}\left|a_{n}\right|\left(1+\frac{1}{n}\right)$, a contradiction.

4.3. The case of the James sequence spaces. Let $p \in(1, \infty)$. We now recall the definition and some basic properties of the James space $\mathcal{J}_{p}$. We refer the reader to $[1$, Section 3.4] and references therein for more details on the classical case $p=2$. The James space $\mathcal{J}_{p}$ is the real Banach space of all sequences $x=(x(n))_{n \in \mathbb{N}}$ of real numbers with finite $p$-variation and satisfying $\lim _{n \rightarrow \infty} x(n)=0$. The space $\mathcal{J}_{p}$ is endowed with the following norm

$$
\|x\|_{\mathcal{J}_{p}}=\sup \left\{\left(\sum_{i=1}^{k-1}\left|x\left(p_{i+1}\right)-x\left(p_{i}\right)\right|^{p}\right)^{1 / p}: 1 \leq p_{1}<p_{2}<\ldots<p_{k}\right\} .
$$

This is the historical example, constructed for $p=2$ by R. C. James, of a quasireflexive Banach space which is isomorphic to its bidual. In fact $\mathcal{J}_{p}^{* *}$ can be seen as the space of all sequences $x=(x(n))_{n \in \mathbb{N}}$ of real numbers with finite $p$-variation, which is $\mathcal{J}_{p} \oplus \mathbb{R} \mathbb{1}$, where $\mathbb{1}$ denotes the constant sequence equal to 1 .

The standard unit vector basis $\left(e_{n}\right)_{n=1}^{\infty}\left(e_{n}(i)=1\right.$ if $i=n$ and $e_{n}(i)=0$ otherwise) is a monotone shrinking basis for $\mathcal{J}_{p}$. Hence, the sequence $\left(e_{n}^{*}\right)_{n=1}^{\infty}$ of the associated coordinate functionals is a basis of its dual $\mathcal{J}_{p}^{*}$. Then the weak topology $\sigma\left(\mathcal{J}_{p}, \mathcal{J}_{p}^{*}\right)$ is 
easy to describe: a sequence $\left(x_{n}\right)_{n=1}^{\infty}$ in $\mathcal{J}_{p}$ converges to 0 in the $\sigma\left(\mathcal{J}_{p}, \mathcal{J}_{p}^{*}\right)$ topology if and only if it is bounded and $\lim _{n \rightarrow \infty} x_{n}(i)=0$ for every $i \in \mathbb{N}$.

For $x \in \mathcal{J}_{p}$, we define $\operatorname{supp} x=\{i \in \mathbb{N}: x(i) \neq 0\}$. For $x, y \in \mathcal{J}_{p}$, we denote: $x \prec y$ whenever max supp $x<\min \operatorname{supp} y$.

The detailed proof of the following proposition can be found in [27, Corollary 2.4]. This a consequence of the following fact: there exists $C \geq 1$ such that $\left\|\sum_{i=1}^{n} x_{i}\right\|_{\mathcal{J}_{p}}^{p} \leq$ $C \sum_{i=1}^{n}\left\|x_{i}\right\|_{\mathcal{J}_{p}}^{p}$, for all $x_{1} \prec \ldots \prec x_{n}$ in $\mathcal{J}_{p}$.

Proposition 4.11. There exists an equivalent norm $|\cdot|$ on $\mathcal{J}_{p}$ such that it has the following property: for any $x, y \in \mathcal{J}_{p}$ such that $x \prec y$, we have that

$$
|x+y|^{p} \leq|x|^{p}+|y|^{p} .
$$

In particular, the modulus of asymptotic uniform smoothness of $\widetilde{\mathcal{J}}_{p}:=\left(\mathcal{J}_{p},|\cdot|\right)$ is $\bar{\rho}_{\widetilde{\mathcal{J}}_{p}}(t) \leq\left(1+t^{p}\right)^{\frac{1}{p}}-1$ for all $t \geq 0$.

There is also a natural weak* topology on $\mathcal{J}_{p}$. Indeed, the summing basis $\left(s_{n}\right)_{n=1}^{\infty}$ $\left(s_{n}(i)=1\right.$ if $i \leq n$ and $s_{n}(i)=0$ otherwise) is a monotone and boundedly complete basis for $\mathcal{J}_{p}$. Thus, $\mathcal{J}_{p}$ is naturally isometric to a dual Banach space: $\mathcal{J}_{p}=X^{*}$ with $X$ being the closed linear span of the biorthogonal functionals $\left(e_{n}^{*}-e_{n+1}^{*}\right)_{n=1}^{\infty}$ in $\mathcal{J}_{p}^{*}$ associated with $\left(s_{n}\right)_{n=1}^{\infty}$. Note that $X=\left\{x^{*} \in \mathcal{J}_{p}^{*}, \sum_{n=1}^{\infty} x^{*}(n)=0\right\}$. Thus, a sequence $\left(x_{n}\right)_{n=1}^{\infty}$ in $\mathcal{J}_{p}$ converges to 0 in the $\sigma\left(\mathcal{J}_{p}, X\right)$ topology if and only if it is bounded and $\lim _{n \rightarrow \infty}\left(x_{n}(i)-x_{n}(j)\right)=0$ for every $i \neq j \in \mathbb{N}$.

The next lemma is classical, we include its proof for completeness.

Lemma 4.12. Let $\left(x_{n}\right)_{n=1}^{\infty}$ be a weak ${ }^{*}$-null sequence in $\mathcal{J}_{p}$. Then, for every $x \in \mathcal{J}_{p}$, we have

$$
\limsup _{n \rightarrow \infty}\left\|x+x_{n}\right\|^{p} \geq\|x\|^{p}+\limsup _{n \rightarrow \infty}\left\|x_{n}\right\|^{p} .
$$

Consequently, the modulus of weak ${ }^{*}$ asymptotic uniform convexity of $\mathcal{J}_{p}$ is given by

$$
\bar{\delta}_{\mathcal{J}_{p}}^{*}(t) \geq\left(1+t^{p}\right)^{\frac{1}{p}}-1 .
$$

Proof. Let $x=(x(n))_{n} \in \mathcal{J}_{p},\left(x_{n}\right)_{n=1}^{\infty}$ be a weak*-null sequence and $\varepsilon>0$ be fixed. Without loss of generality, we may and do assume that $\|x\|=1$. Let $\left(p_{i}\right)_{i=1}^{N} \subset \mathbb{N}$ be an increasing family such that

$$
1-\varepsilon \leq\left(\sum_{i=1}^{N-1}\left|x\left(p_{i+1}\right)-x\left(p_{i}\right)\right|^{p}\right)^{1 / p}
$$

Then there exists $N_{1}>p_{N}$ such that for any increasing finite sequence $\left(q_{i}\right)_{i=1}^{k}$ with $q_{1} \geq N_{1}$,

$$
\left(\sum_{i=1}^{k-1}\left|x\left(q_{i+1}\right)-x\left(q_{i}\right)\right|^{p}\right)^{1 / p} \leq \eta
$$

where $0<\eta<\varepsilon$ will be precisely chosen at the end of the proof. Now, since $\left(x_{n}\right)_{n=1}^{\infty}$ is a weak ${ }^{*}$-null sequence, it is bounded by some $M>0$ and there exists $N_{2} \in \mathbb{N}$ large enough so that for every $n \geq N_{2}$ and for any increasing finite sequence $\left(r_{i}\right)_{i=1}^{l}$ with $r_{l}<N_{1}$ :

$$
\sum_{i=1}^{l-1}\left|x_{n}\left(r_{i+1}\right)-x_{n}\left(r_{i}\right)\right|^{p} \leq \frac{\eta}{3} \quad \text { and } \quad\left|x_{n}\left(r_{l}\right)-x_{n}\left(N_{1}\right)\right|^{p} \leq \eta^{\prime},
$$

where $\eta^{\prime}$ is chosen so that $\|\left. x|-| y\right|^{p} \geq|x|^{p}-\frac{\eta}{3}$ for every $x \in[0, M]$ and $y \in\left[0, \eta^{\prime}\right]$ (the map $f(x, y)=|x-y|^{p}$ is Lipschitz on $[0, M] \times[0, M]$, so $\eta^{\prime}=\min \left\{\frac{\eta}{3 \operatorname{Lip}(f)}, M\right\}$ 
does the work). Note also that in such a case one also has

$$
\begin{aligned}
\left|x_{n}(r)-x_{n}\left(N_{1}\right)\right|^{p} & \geq|| x_{n}(r)-x_{n}\left(r_{l}\right)|-| x_{n}\left(r_{l}\right)-x_{n}\left(N_{1}\right)||^{p} \\
& \geq\left|x_{n}(r)-x_{n}\left(r_{l}\right)\right|^{p}-\frac{\eta}{3}
\end{aligned}
$$

for any $r \geq N_{1}$.

Now, let $n \geq N_{2}$. It follows from the above estimates that we may find a fixed increasing sequence $\left(q_{i}\right)_{i=1}^{k}$ with $q_{1} \geq N_{1}$ and

$$
\sum_{i=1}^{k-1}\left|x_{n}\left(q_{i+1}\right)-x_{n}\left(q_{i}\right)\right|^{p} \geq\left\|x_{n}\right\|^{p}-\eta
$$

Note that $p_{N}<N_{1} \leq q_{1}$ by construction so that

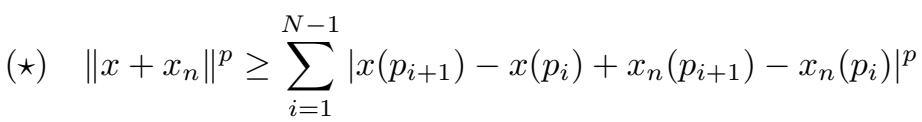

$$
\begin{aligned}
& +\sum_{i=1}^{k-1}\left|x\left(q_{i+1}\right)-x\left(q_{i}\right)+x_{n}\left(q_{i+1}\right)-x_{n}\left(q_{i}\right)\right|^{p} .
\end{aligned}
$$

But

$$
\begin{aligned}
\left(\sum_{i=1}^{N-1}\left|x\left(p_{i+1}\right)-x\left(p_{i}\right)+x_{n}\left(p_{i+1}\right)-x_{n}\left(p_{i}\right)\right|^{p}\right)^{\frac{1}{p}} & \left.\geq\left(\sum_{i=1}^{N-1}\left|x\left(p_{i+1}\right)-x\left(p_{i}\right)\right|\right)^{p}\right)^{\frac{1}{p}}-\varepsilon \\
& \geq 1-2 \varepsilon
\end{aligned}
$$

which implies that $\sum_{i=1}^{N-1}\left|x\left(p_{i+1}\right)-x\left(p_{i}\right)+x_{n}\left(p_{i+1}\right)-x_{n}\left(p_{i}\right)\right|^{p} \geq 1-2 p \varepsilon$.

Similarly, we obtain that

$$
\sum_{i=1}^{N-1}\left|x\left(q_{i+1}\right)-x\left(q_{i}\right)+x_{n}\left(q_{i+1}\right)-x_{n}\left(q_{i}\right)\right|^{p} \geq\left\|x_{n}\right\|^{p}-2 p M \eta .
$$

Finally, if we chose $\eta<\min \left(\varepsilon, \frac{\varepsilon}{M}\right)$, we obtain from $(\star)$ the following last estimate

$$
\left\|x+x_{n}\right\|^{p} \geq 1+\left\|x_{n}\right\|^{p}-4 p \varepsilon .
$$

As an application of Theorem 3.1 and the two previous lemmata, we obtain the following corollary.

Corollary 4.13. If $1<p \leq q<\infty$ then $\left(\mathcal{J}_{p}, \widetilde{\mathcal{J}}_{q}\right)$ has the weak $k^{*}$-to-weak $k^{*} M P$.

We now turn to an application of Lemma 3.6 and Theorem 3.8.

Corollary 4.14. If $1<q<p<\infty$ then every bounded operator from $\mathcal{J}_{p}$ to $\mathcal{J}_{q}$ is compact. In particular, the pair $\left(\mathcal{J}_{p}, \mathcal{J}_{q}\right)$ has the weak*-to-weak* $M P$.

Proof. Since $\bar{\rho} \widetilde{\mathcal{J}}_{p}(t) \leq\left(1+t^{p}\right)^{1 / p}-1, \bar{\delta}_{\mathcal{J}_{q}}^{*}(t) \geq\left(1+t^{q}\right)^{1 / q}-1$ and $\bar{\delta}_{\mathcal{J}_{q}}(t) \geq \bar{\delta}_{\mathcal{J}_{q}}^{*}(t)$, we obtain from Lemma 3.6 that every bounded operator from $\widetilde{\mathcal{J}}_{p}$ to $\mathcal{J}_{q}$ is compact. Since $\widetilde{\mathcal{J}}_{p}$ and $\mathcal{J}_{p}$ are isomorphic, this yields the same conclusion for every bounded operator from $\mathcal{J}_{p}$ to $\mathcal{J}_{q}$. To conclude, Theorem 3.8 (2) provides the fact that the pair $\left(\mathcal{J}_{p}, \mathcal{J}_{q}\right)$ has the weak*-to-weak ${ }^{*}$ MP.

Having in mind the previous corollaries, it is quite natural to wonder the following:

Question 4.15. Does the pair $\left(\mathcal{J}_{p}, \mathcal{J}_{q}\right)$ has the weak*-to-weak*MP for $1<p \leq$ $q<\infty$ ? 
Remark 4.16. Quite surprisingly, the pair $\left(\mathcal{J}_{2}, \mathbb{R}\right)$ fails the $\mathrm{W}^{*} \mathrm{MP}$ (but has the weak*-to-normMP thanks to Corollary 4.13). Indeed, consider $T: \mathcal{J}_{2} \rightarrow \mathbb{R}$ be the linear operator given by

$$
\forall x=(x(j))_{j=1}^{\infty} \in \mathcal{J}_{2}, \quad T x:=\frac{-x(1)}{2}+\sum_{j=2}^{\infty} \frac{x(j)}{j^{2}} .
$$

That is, $T=\left(\frac{-1}{2}, \frac{1}{2^{2}}, \frac{1}{3^{2}}, \ldots\right) \in \mathcal{J}_{2}^{*}$. Note that $T$ is not weak* continuous since $T\left(s_{n}\right) \not \rightarrow 0$ while the summing basis $\left(s_{n}\right)$ is weak*-null. Since the standard unit vector basis $\left(e_{n}\right)_{n=1}^{\infty}$ is monotone and shrinking, it follows that for every $x^{* *} \in \mathcal{J}_{2}^{* *}$, $\left\|x^{* *}\right\|_{\mathcal{J}_{2}^{* *}}=\sup _{n}\left\|\left(x^{* *}\left(e_{1}^{*}\right), x^{* *}\left(e_{2}^{*}\right), \ldots, x^{* *}\left(e_{n}^{*}\right), 0, \ldots\right)\right\|_{\mathcal{J}_{2}}$ (see e.g. Proposition 4.14 in [13]. Thus, it not hard to prove that any norming functional $x^{* *}$ for $T$ is of the form $x^{* *}=\frac{1}{\sqrt{\left(t_{2}-t_{1}\right)^{2}+t_{2}^{2}}}\left(t_{1}, t_{2}, t_{2}, \ldots\right)$. Consequently, in order to maximize $T\left(x^{* *}\right)$ it suffices to maximize the function

$$
g\left(t_{1}, t_{2}\right)=\frac{\frac{-t_{1}}{2}+t_{2}\left(\frac{\pi^{2}}{6}-1\right)}{\sqrt{\left(t_{2}-t_{1}\right)^{2}+t_{2}^{2}}}=\frac{\frac{-1}{2} \frac{t_{1}}{t_{2}}+\left(\frac{\pi^{2}}{6}-1\right)}{\sqrt{\left(1-\frac{t_{1}}{t_{2}}\right)^{2}+\left(\frac{t_{1}}{t_{2}}\right)^{2}}} .
$$

We set $t=\frac{t_{1}}{t_{2}}$ and we are now looking for the maximum of the following one variable map:

$$
f(t)=\frac{\frac{-1}{2} t+\left(\frac{\pi^{2}}{6}-1\right)}{\sqrt{(1-t)^{2}+t^{2}}} .
$$

A basic study of the map $f$ shows that it attains its maximum at $t_{\max }=\frac{\pi^{2}-9}{2 \pi^{2}-15}$ (and $\left.f\left(t_{\max }\right) \simeq 0.66\right)$. To conclude, fix $t_{1}, t_{2} \in \mathbb{R}$ such that $\frac{t_{1}}{t_{2}}=t_{\max }$ and $\sqrt{\left(t_{2}-t_{1}\right)^{2}+t_{2}^{2}}=1$. Now for every $n \in \mathbb{N}$ let $x_{n} \in \mathcal{J}_{2}$ be such that $x_{n}(1)=t_{1}$, $x_{n}(2)=\ldots=x_{n}(n)=t_{2}$ and $x_{n}(j)=0$ whenever $j>n$ (that is, $x_{n}=$ $\left.\left(t_{1}, t_{2}, \ldots, t_{2}, 0, \ldots\right)\right)$. Then $\left(x_{n}\right)_{n=1}^{\infty}$ is a normalized maximizing sequence for $T$ which is not weak*-null. However, it is easy to see that $T$ does not attains its norm (as every element in $\mathcal{J}_{2}$ is a sequence that converges to 0 ).

4.4. Orlicz spaces. Given an Orlicz function $\varphi:[0,+\infty) \rightarrow[0,+\infty)$ (that is, $\varphi$ is a continuous convex unbounded function with $\varphi(0)=0$ ), the Orlicz sequence space $\ell_{\varphi}$ is the space of all real sequences $x=\left(x_{n}\right)_{n=1}^{\infty}$ such that $\sum_{n=1}^{\infty} \varphi\left(\left|x_{n}\right| / \lambda\right)<\infty$. It is a Banach space when equipped with the Luxemburg norm:

$$
\|x\|_{\varphi}=\inf \left\{\lambda>0: \sum_{n=1}^{\infty} \varphi\left(\left|x_{n}\right| / \lambda\right) \leq 1\right\} .
$$

The closed linear span of $\left\{e_{n}: n \in \mathbb{N}\right\}$ in $\ell_{\varphi}$ is denoted $h_{\varphi}$. The space $h_{\varphi}$ coincides with $\ell_{\varphi}$ precisely if $\varphi$ satisfies the $\Delta_{2}$ condition at zero, i.e. $\lim \sup _{t \rightarrow 0} \varphi(2 t) / \varphi(t)<$ $\infty$. The space $h_{\varphi}\left(\right.$ or $\left.\ell_{\varphi}\right)$ is reflexive if and only if both $\varphi$ and $\varphi^{*}$ satisfy the $\Delta_{2}$ condition at zero, where $\varphi *(t)=\sup \{s t-\varphi(s)\}$ is the convex conjugate of $\varphi$. The Boyd indices of an Orlicz function $\varphi$ are defined as follows:

$$
\alpha_{\varphi}=\sup \left\{p>0: \sup _{0<u, t \leq 1} \frac{\varphi(t u)}{\varphi(u) t^{p}}<\infty\right\}, \quad \beta_{\varphi}=\inf \left\{p>0: \inf _{0<u, t \leq 1} \frac{\varphi(t u)}{\varphi(u) t^{p}}>0\right\} .
$$

It is known that $\beta_{\varphi}<\infty$ precisely if $\varphi$ satisfies the $\Delta_{2}$ condition at 0 . The asymptotic moduli of the space $h_{\varphi}$ is linked to the Boyd indices: $h_{\varphi}$ is AUS (resp. AUC) if and only if $\alpha_{\varphi}>1$ (resp. $\beta_{\varphi}<\infty$ ). Indeed, $h_{\varphi}$ is AUS if and only if $\alpha_{\varphi}>1$, and $\alpha_{\varphi}$ is the supremum of the numbers $\alpha$ such that $\bar{\rho}_{h_{\varphi}}$ has power type $\alpha$ 
[16]. In addition, $\beta_{\varphi}$ is the infimum of the numbers $\beta$ such that $\bar{\delta}_{h_{\varphi}}$ has power type $\beta[7]$.

In order to apply Theorem 3.1 to the case of Orlicz spaces we need an estimation of $\bar{\rho}_{h_{\varphi}}(t)$ and $\bar{\delta}_{h_{\varphi}}(t)$ for all $t>0$. To this end, consider the following indices:

$$
\begin{aligned}
& p_{\varphi}=\sup \left\{p>0: u^{-p} \varphi(u) \text { is non-decreasing for all } 0<u \leq \varphi^{-1}(1)\right\} \\
& q_{\varphi}=\inf \left\{p>0: u^{-p} \varphi(u) \text { is non-increasing for all } 0<u \leq \varphi^{-1}(1)\right\}
\end{aligned}
$$

Clearly $1 \leq p_{\varphi} \leq q_{\varphi} \leq \infty$. Moreover, $\varphi$ (resp. $\varphi^{*}$ ) satisfies $\Delta_{2}$ condition at 0 if and only if $q_{\varphi}<\infty$ (resp. $p_{\varphi}>1$ ), see [25] or [10]. Delphech [10] showed that if $q_{\varphi}<\infty$ then

$$
\left(1+t^{q_{\varphi}}\right)^{1 / q_{\varphi}}-1 \leq \bar{\delta}_{h_{\varphi}}(t)
$$

for all $t \in(0,1]$, but actually the proof works for all $t>0$. Analogously, one can show that

for all $t>0$.

$$
\bar{\rho}_{h_{\varphi}}(t) \leq\left(1+t^{p_{\varphi}}\right)^{1 / p_{\varphi}}-1
$$

Remark 4.17. In general $\left[\alpha_{\varphi}, \beta_{\varphi}\right] \subset\left[p_{\varphi}, q_{\varphi}\right]$, but the inclusion may be strict [25]. Thus the previous inequalities are not tight. Also, recall that, given any $p \in\left[\alpha_{\varphi}, \beta_{\varphi}\right]$, the space $h_{\varphi}$ contains almost isometric copies of $\ell_{p}$. Thus $\bar{\delta}_{\ell_{\varphi}}(t) \leq\left(1+t^{\alpha_{\varphi}}\right)^{1 / \alpha_{\varphi}}-1$ and $\bar{\rho}_{\ell_{\varphi}}(t) \geq\left(1+t^{\beta_{\varphi}}\right)^{1 / \beta_{\varphi}}-1$ for all $t>0$.

Corollary 4.18. Let $\varphi, \psi$ be Orlicz functions. Assume that $h_{\varphi}, h_{\psi}$ are reflexive and $q_{\varphi} \leq p_{\psi}$. Then the pair $\left(h_{\varphi}, h_{\psi}\right)$ has the WMP.

Remark 4.19. Given Orlicz functions $\varphi, \psi$, if $\alpha_{\varphi}>\beta_{\psi}$, then every operator from $h_{\varphi}$ to $h_{\varphi}=\ell_{\psi}$ is compact [6], and so $\left(h_{\varphi}, h_{\psi}\right)$ has the WMP.

4.5. Remarks about the pair $\left(L_{p}, L_{q}\right)$. In what follows, $L_{p}$ stands for $L_{p}([0,1])$ with $1<p<\infty$. It is known (see [26] at page 117 for instance) that if $X=L_{p}$ then there exist constants $C_{1}(p), C_{2}(p)$ such that for $1<p<2$ we have

and for $2<p<\infty$ we have that

$$
\begin{aligned}
C_{1}(p) t^{2} & \leq \bar{\delta}_{X}(t) \leq(p-1) t^{2} \\
\frac{1}{p} t^{p} & \leq \bar{\rho}_{X}(t) \leq \frac{2}{p} t^{p}
\end{aligned}
$$

$$
\begin{aligned}
C_{1}(p) t^{p} & \leq \bar{\delta}_{X}(t) \leq \frac{1}{p} t^{p} \\
(p-1) t^{2} & \leq \bar{\rho}_{X}(t) \leq C_{2}(p) t^{2} .
\end{aligned}
$$

Consequently, for $1<p<q<\infty$, we cannot apply Theorem 3.1 to prove that the pair $\left(L_{p}, L_{q}\right)$ has the WMP. Nevertheless, replacing the weak topology by the topology $\tau_{m}$ of convergence in measure, we can apply similar techniques to prove the following related results.

Proposition 4.20. Let $1<p<q<\infty$ an let $T: L_{p} \rightarrow L_{q}$ be a bounded operator which is $\tau_{m}$-to- $\tau_{m}$ continuous. If there exists a maximizing sequence $\left(x_{n}\right)_{n} \subset L_{p}$ for $T$ which $\tau_{m}$-converges to some $x \neq 0$, then $T$ attains its norm at $x$.

Proof. The proof follows the same lines as in Theorem 3.1. In fact, $L_{p}$ has the following property (see [20] e.g.): if $\left(x_{n}\right)_{n} \subset L_{p}$ converges to 0 in measure, then for every $x \in L_{p}$

(*) $\quad \limsup _{n}\left\|x+x_{n}\right\|=\left(\|x\|^{p}+\underset{n}{\limsup }\left\|x_{n}\right\|^{p}\right)^{1 / p}$. 
Let $T: L_{p} \rightarrow L_{q}$ be a bounded operator which is $\tau_{m}$-to- $\tau_{m}$ continuous and let $\left(x_{n}\right)_{n} \subset L_{p}$ be a maximizing sequence for $T$ which $\tau_{m}$-converges to some $x \neq 0$. Without loss of generality, we may assume that $T$ has norm 1 . Since $T$ is $\tau_{m}$-to- $\tau_{m}$ continuous, we have that $T x_{n} \underset{n}{\stackrel{\tau_{m}}{\longrightarrow}} T x$. Using (*), we thus have the following estimates:

$$
\begin{aligned}
1 & =\|T\|=\lim _{n}\left\|T x_{n}\right\|=\lim _{n}\left\|T x+T x_{n}-T x\right\| \\
& =\left(\|T x\|^{q}+\lim _{n}\left\|T x_{n}-T x\right\|^{q}\right)^{\frac{1}{q}} \\
& \leq\left(\|T x\|^{q}+\lim _{n}\left\|x_{n}-x\right\|^{q}\right)^{\frac{1}{q}} \\
& \leq\left(\|T x\|^{p}+\lim _{n}\left\|x_{n}-x\right\|^{p}\right)^{\frac{1}{p}} \\
& =\left(\|T x\|^{p}+1-\|x\|^{p}\right)^{\frac{1}{p}}
\end{aligned}
$$

Therefore, we deduce that $\|T x\| \geq\|x\|$, which finishes the proof.

Now the lack of $\tau_{m}$-compactness of the unit ball of $L_{p}$ forces us to consider pairs $\left(X, L_{q}\right)$ where $X$ is a subspace of $L_{p}$ whose unit ball $B_{X}$ is $\tau_{m}$-compact. For instance, it is proved in [20] that $X$ embeds almost isometrically into $\ell_{p}$ if and only if $B_{X}$ is compact for the topology inherited by the $L_{1}$-norm; for those $X, B_{X}$ is in particular $\tau_{m}$-compact. To the best of our knowledge, such subspaces haven't been characterized.

Corollary 4.21. Let $1<p<q<\infty$. If $X$ is a subspace of $L_{p}$ such that $B_{X}$ is $\tau_{m}$-compact, then the pair $\left(X, L_{q}\right)$ has the $\tau_{m}$-to- $\tau_{m} M P$.

Let us briefly discuss the case when $1<q<p<\infty$. For the pair $\left(\ell_{p}, \ell_{q}\right)$, this was handled by Pitts Theorem (or more generally by Theorem 3.8) saying that every operator from $\ell_{p}$ to $\ell_{q}$ is compact. This approach fails for $\left(L_{p}, L_{q}\right)$. Indeed, H. P. Rosenthal characterized in [29, Theorem A.2] when every operator from $L_{p}(\mu)$ to $L_{q}(\nu)$ is compact. That is never the case for $L_{p}[0,1]$ and $L_{q}[0,1]$. However, if $q<p, 1 \leq q<2$, then every operator from $L_{p}[0,1]$ to $\ell_{q}$ is compact, and if $q<p$ and $2<p$ then every operator from $\ell_{p}$ to $L_{q}$ is compact.

\section{ACKNOWLedgments}

Part of this work was carried out during a visit of the two named authors in Murcia (Spain) in January 2020. They are deeply grateful to Matías Raja and the "Universidad de Murcia" for the hospitality and excellent working conditions there. The authors would like to thank Richard M. Aron for useful conversations. The first author is supported in part by the grants MTM2017-83262-C2-2-P and Fundación Séneca Región de Murcia 20906/PI/18 also supported by a postdoctoral grant from Fundación Séneca.

\section{REFERENCES}

[1] F. Albiac and N. J. Kalton, Topics in Banach space theory, vol. 233 of Graduate Texts in Mathematics, Springer, [Cham], second ed., 2016. With a foreword by Gilles Godefory.

[2] R. J. Aliaga And A. J. GuiraO, On the preserved extremal structure of Lipschitz-free spaces, Studia Math., 245 (2019), pp. 1-14.

[3] R. J. Aliaga, E. Pernecká, C. Petitjean, and A. Procházka, Supports in Lipschitz-free spaces and applications to extremal structure, J. Math. Anal. Appl., 489 (2020), pp. 124128, 14 .

[4] C. D. Aliprantis and K. C. Border, Infinite dimensional analysis, Springer, Berlin, third ed., 2006. A hitchhiker's guide.

[5] R. M. Aron, D. García, D. Pellegrino, and E. V. Teixeira, Reflexivity and nonweakly null maximizing sequences, Proc. Amer. Math. Soc., 148 (2020), pp. 741-750. 
[6] E. A. Ausekle And È. F. Oya, Pitt's theorem for Lorentz and Orlicz sequence spaces, Mat. Zametki, 61 (1997), pp. 18-25.

[7] L. Borel-Mathurin, Isomorphismes non linéaires entre espaces de Banach, PhD thesis, Université Paris 6, 2010.

[8] B. M. Braga, Asymptotic structure and coarse Lipschitz geometry of Banach spaces, Studia Math., 237 (2017), pp. 71-97.

[9] R. M. Causey and G. Lancien, Prescribed Szlenk index of separable Banach spaces, Studia Math., 248 (2019), pp. 109-127.

[10] S. Delpech, Asymptotic uniform moduli and Kottman constant of Orlicz sequence spaces, Rev. Mat. Complut., 22 (2009), pp. 455-467.

[11] J. Diestel, A survey of results related to the Dunford-Pettis property, in Proceedings of the Conference on Integration, Topology, and Geometry in Linear Spaces (Univ. North Carolina, Chapel Hill, N.C., 1979), vol. 2 of Contemp. Math., Amer. Math. Soc., Providence, R.I., 1980, pp. $15-60$.

[12] S. J. Dilworth, D. Kutzarova, G. Lancien, and N. L. Randrianarivony, Equivalent norms with the property $(\beta)$ of Rolewicz, Rev. R. Acad. Cienc. Exactas Fís. Nat. Ser. A Mat. RACSAM, 111 (2017), pp. 101-113.

[13] M. Fabian, P. Habala, P. Hájek, V. Montesinos, and V. Zizler, Banach space theory, CMS Books in Mathematics/Ouvrages de Mathématiques de la SMC, Springer, New York, 2011. The basis for linear and nonlinear analysis.

[14] L. García-Lirola, C. Petitjean, A. Procházka, and A. Rueda Zoca, Extremal structure and duality of Lipschitz free spaces, Mediterr. J. Math., 15 (2018), pp. Art. 69, 23.

[15] G. Godefroy, N. Kalton, And G. Lancien, Subspaces of $c_{0}(\mathbf{N})$ and Lipschitz isomorphisms, Geom. Funct. Anal., 10 (2000), pp. 798-820.

[16] R. Gonzalo, J. A. Jaramillo, and S. L. Troyanski, High order smoothness and asymptotic structure in Banach spaces, J. Convex Anal., 14 (2007), pp. 249-269.

[17] W. B. Johnson, J. Lindenstrauss, D. Preiss, and G. Schechtman, Almost Fréchet differentiability of Lipschitz mappings between infinite-dimensional Banach spaces, Proc. London Math. Soc. (3), 84 (2002), pp. 711-746.

[18] N. Kalton, Uniform homeomorphisms of Banach spaces and asymptotic structure, Trans. Amer. Math. Soc., 365 (2013), pp. 1051-1079.

[19] N. J. Kalton, Spaces of Lipschitz and Hölder functions and their applications, Collect. Math., 55 (2004), pp. 171-217.

[20] N. J. Kalton And D. Werner, Property $(M), M$-ideals, and almost isometric structure of Banach spaces, J. Reine Angew. Math., 461 (1995), pp. 137-178.

[21] H. Knaust, E. Odell, and T. Schlumprecht, On asymptotic structure, the Szlenk index and UKK properties in Banach spaces, Positivity, 3 (1999), pp. 173-199.

[22] J. Kover, Compact perturbations and norm attaining operators, Quaest. Math., 28 (2005), pp. 401-408.

[23] G. LAnCIEn, A survey on the Szlenk index and some of its applications, RACSAM. Rev. R. Acad. Cienc. Exactas Fís. Nat. Ser. A Mat., 100 (2006), pp. 209-235.

[24] J. Lindenstrauss and L. Tzafriri, Classical Banach spaces. I, Springer-Verlag, Berlin-New York, 1977. Sequence spaces, Ergebnisse der Mathematik und ihrer Grenzgebiete, Vol. 92.

[25] L. Maligranda, Indices and interpolation, Dissertationes Math. (Rozprawy Mat.), 234 (1985), p. 49.

[26] V. D. Milman, Geometric theory of Banach spaces. II. Geometry of the unit ball, Uspehi Mat. Nauk, 26 (1971), pp. 73-149.

[27] F. Netillard, Coarse Lipschitz embeddings of James spaces, Bull. Belg. Math. Soc. Simon Stevin, 25 (2018), pp. 71-84.

[28] D. Pellegrino And E. V. Teixeira, Norm optimization problem for linear operators in classical Banach spaces, Bull. Braz. Math. Soc. (N.S.), 40 (2009), pp. 417-431.

[29] H. P. Rosenthal, On quasi-complemented subspaces of Banach spaces, with an appendix on compactness of operators from $L^{p}(\mu)$ to $L^{r}(\nu)$, J. Functional Analysis, 4 (1969), pp. 176-214.

(L. García-Lirola) Department of Mathematical Sciences, Kent State University, Kent, $\mathrm{OH} 44242$, USA

E-mail address: lgarcial@kent.edu

(C. Petitjean) Lama, Univ Gustave Eiffel, Upem, Univ Paris Est Creteil, CNRS, F-77447, Marne-LA-VAllÉE, France

E-mail address: colin.petitjean@u-pem.fr 